\title{
Strength Tests and Quality Evaluation of Core Samples of Cement Mixing Piles
}

\author{
Ben-Jiao ZHANG ${ }^{1, a}$, Bin HUANG ${ }^{1,2, b}$, Jun RAO ${ }^{1, c}$, Xu-Dong FU ${ }^{1, d}$ \\ ${ }^{1}$ College of Civil and Architecture Engineering, Wuhan University, Wuhan, Hubei, 430072 \\ ${ }^{2}$ Changjiang River Scientific Research Institute, Key Laboratory of Geotechnical Mechanics and \\ Engineering of the Ministry of Water Resources ,Wuhan, Hubei, 430010 \\ azhangbjwhu@163.com, ${ }^{\mathrm{b}}$ cucumberhb@163.com, ${ }^{\mathrm{c}} \mathrm{jxwnrj@139.com,}{ }^{\mathrm{d} x d f u @ w h u . e d u . c n}$
}

Keywords: Cement Mixing Pile, Strength Property, Quality Evaluation, Standard Value, Variable Coefficient, Modulus Ratio.

\begin{abstract}
In order to study strength and evaluate its quality of cement-soil in on-site construction process, unconfined compressive tests and triaxial CU shear tests were carried out on cement-soil core samples. The analysis of effects of cement mixing content, water cement ratio and lifting speed on strength and deformation properties of cement-soil was made. The results show that with the improvement of cement mixing ratio, the strength of cement-soil core samples is enhanced obviously, deformation modulus increases significantly, but the failure strain decreases, brittleness increases. And in the same conditions of cement mixing ratio, the strength of cement soil is higher when water cement ratio is 1.0 than that when water cement ratio is 0.8 . And as the lifting speed increases, the density and strength of cement soil core samples are significantly reduced. This paper introduces the concepts of standard value, variable coefficient and modulus ratio, which can evaluate the density of cement soil core samples, unconfined compressive strength and the triaxial $\mathrm{CU}$ shear strength. This paper gives rational advice and is certain guide significance for practical projects.
\end{abstract}

\section{Introduction}

In China's coastal areas, the soft soil distributes widely on both sides of inland river and lake areas, building roads or constructions in these areas need to reinforce the soft soil[1]. Since construction machinery of cement-soil pile was successfully developed and applied to engineering in the last century, because soft soil reinforcement technology of cement-soil pile has the advantages of maximizing the use of the foundation soil, less effects on the existing buildings; no vibration, noise and pollution during construction; lower cost and shorter construction period by comparing to other methods, etc. it has been rapidly and widely used in engineering construction such as highway, railway, water conservancy, the municipal, construction projects and so on[2].

Strength and deformation properties of cement soil have a strong impact on carrying capacity and deformation behavior of cement mixing pile. Many scholars have carried on research to strength and deformation of cement soil and have made a great many important results [3-7]. However, these cement soil samples were made of the soil which was dug at the scene in the test chamber and were tested after curing. The processes and the curing condition are quite different from the field condition. Many scholars have carried on research to the homogeneity of on-site cement mixing piles [8], the destabilization from construction [9], the variability of cement soil strength [10]. There are also some scholars carrying on indoor and outdoor comparative research to the strength of cement soil $[11,12]$. On the basis of previous academic studies, this paper use drilling core samples and carry out unconfined compressive tests and triaxial CU shear tests on multiple sets of cement-soil core samples. The analysis of effects of cement mixing content, water cement ratio and lifting speed on strength and deformation properties of cement-soil was made. This paper introduces the concepts of standard value, variable coefficient and modulus ratio, evaluates the density of cement soil core samples, and comprehensively evaluates the strength and deformation properties of cement-soil in on-site construction process. 


\section{Experiment Scheme}

\section{Sample Preparation}

In a project, cement mixing piles go through different stratums including sand layer, clay stratum, etc. In this paper, core samples are drilled from the part of cement mixing pile in sand layer, on which indoor strength and deformation tests are carried out. Representative cement-soil core samples in sand layer are selected, whose age is over 28 days, and were cut into samples whose height-diameter ratio is $2: 1$. And the ends of samples were grinded, the qualities of sample were weighed and the sizes of sample were measured according to Standard for test methods of engineering rock mass [13].

\section{Test Equipment}

Unconfined compressive tests and triaxial CU tests to the cement-soil core samples were carried out on SY250-type static triaxial compression apparatus. The test equipment is shown in Figure 1. The mainly technical indicators of this test equipment are: the samples size is $\Phi 101 \mathrm{~mm} \times 200 \mathrm{~mm}$, the maximum axial load is $300 \mathrm{kN}$, the maximum confining pressure is $1000 \mathrm{kPa}$. The measurement accuracy of volumetric change is $0.1 \mathrm{~mL}$. When it is reinforced sandy soil by cement, it often manifests as shear dilatation, the volumetric changes of samples were measured by the external volumetric change measuring equipment. Calculate the volumetric changes of samples by measuring the quality change of the water in the pressure chamber of volumetric change measuring equipment.
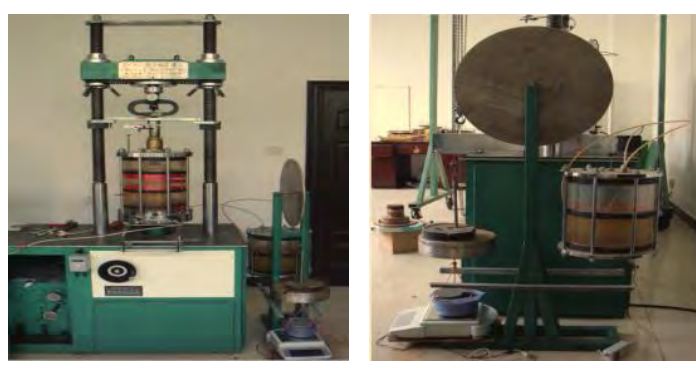

Fig. 1 SY250-type Static Triaxial Compression Apparatus

\section{Experiment Scheme}

After cured, samples are installed and prepared to be tested. If it is unconfined compressive test, then samples will be tested directly. After installed, samples are loaded with confining pressure and isobaric consolidation is carried out when it is triaxial CU shear test. When the external volumetric change keeps constant, the consolidation is stable, after consolidation is over, the axial load is applied to shear. During shearing keep the drain valve open. Record the test data once manually when the axial displacement change $0.1 \mathrm{~mm}$ in the shearing process. Density tests, unconfined compressive tests and CU shear tests whose confining pressure was $100 \mathrm{kPa}$ were carried out on all the core samples whose stake mark was from D-1 to D-8.

\section{Test Results and Rules}

\section{Density Test}

Results of density tests on cement-soil core samples are shown in Table 1. From the test results we can see that there is certain discreteness among the densities of cement-soil core samples. But the overall performance of results is that densities of core samples are greater when the cement mixing ratio is $20 \%$ than the ones when the cement mixing ratio is $15 \%$. The variable coefficient of the densities of cement-soil core samples is greater when the lifting speed is $0.94 \mathrm{~m} / \mathrm{min}$ than the one when the lifting speed is $0.59 \mathrm{~m} / \mathrm{min}$. To analyze from the aspect of density, the lifting speed of construction has some influence on the homogeneity of cement mixing piles. Slower speed is more beneficial to of the homogeneity the pile. 
Tab. 1 Results of Density Tests on Cement-soil Core Samples

\begin{tabular}{cccccc}
\hline Stake mark & Water cement & Cement mixing & Lifting speed & Natural & Variable \\
\hline D-1 & 0.8 & 15 & 0.59 & $\frac{1.71 \sim 1.85}{1.77(8)}$ & 0.0400 \\
D-2 & 1 & 15 & 0.59 & $\frac{1.46 \sim 1.65}{1.56(8)}$ & 0.0497 \\
D-3 & 0.8 & 20 & 0.59 & $\frac{1.65 \sim 1.93}{1.85(1)}$ & 0.0492 \\
D-4 & 0.8 & 15 & 0.94 & $\frac{1.54 \sim 1.91}{1.67(10)}$ & 0.0759 \\
D-5 & 1 & 20 & 0.59 & $\frac{1.69 \sim 1.94}{1.83(10)}$ & 0.0416 \\
D-6 & 1 & 15 & 0.94 & $\frac{1.74 \sim 1.89}{1.83(10)}$ & 0.0302 \\
D-7 & 0.8 & 20 & 0.94 & $\frac{1.66 \sim 1.99}{1.82(10)}$ & 0.0687 \\
D-8 & 1 & 20 & 0.94 & $\frac{1.63 \sim 2.10}{1.94(10)}$ & 0.0769 \\
\hline
\end{tabular}

minimu $\sim$ maximum value

Annotation: average number (class number)

Unconfined Compressive Test

Tab. 2 Results of Unconfined compression Tests on Cement-soil Core Samples

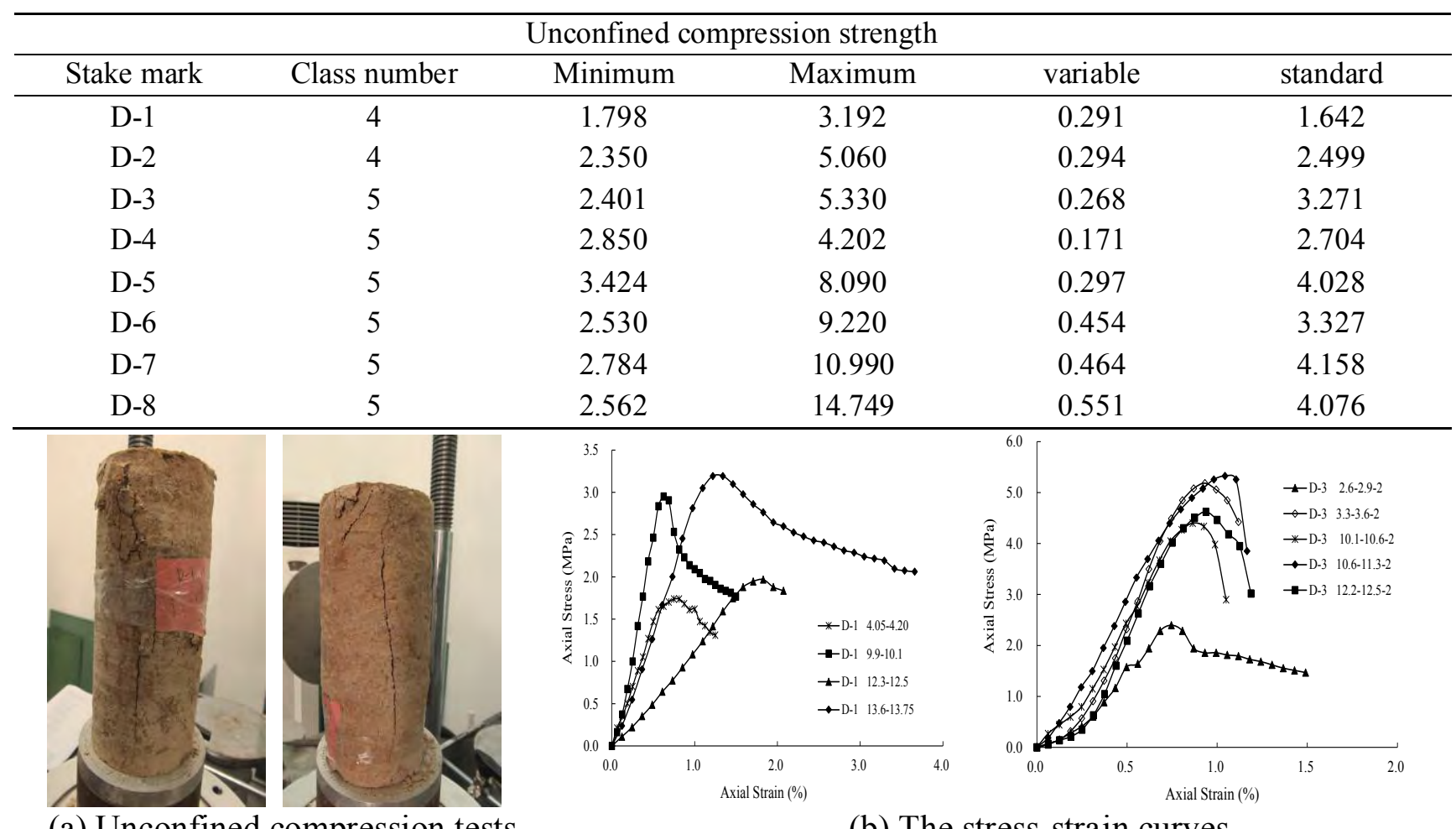

(a) Unconfined compression tests

(b) The stress-strain curves

Fig. 2 Unconfined Compression Tests

4 to 5 groups of unconfined compression tests were carried out on cement-soil core samples of each stake mark according to Specification of soil test (SL237-020-1999). The rate of the test is $1.20 \mathrm{~mm} / \mathrm{min}$. The results are shown in Table 2 and the failure modes are shown in Figure 2(a). Test results of unconfined compressive tests on cement-soil core samples of D-1 and D-3 are shown in Figure 2(b). The figure and the table show that:

(1)Cement-soil core samples are of brittle tension failure in the unconfined compressive tests, whose tension cracks spread mainly along the vertical direction of the samples. And the stress-strain relations show strain softening. The failure strains are at a range from $0.52 \%$ to $1.85 \%$.

(2)Results of modulus present large discreteness because the failure strains in tests are very small. Besides, the accuracy of equipment and human factors will have a great impact on the results. The 
reliability of results in indoor tests is better. It is an objective reality that the strengths of the on-site construction piles are heterogeneous and it is difficult to achieve the same homogeneity with the indoor mix proportion test.

\section{Triaxial CU Shear Test}

The triaxial CU shear tests were conducted according to Specification of soil test (SL237-020-1999). 4 to 5 groups of triaxial CU shear tests whose confining pressure was $100 \mathrm{kPa}$ was carried out on cement-soil core samples of each stake mark. Samples are loaded with confining pressure after installed. Maintain the confining pressure for 30 minutes and then start to shear with the rate of $1.20 \mathrm{~mm} / \mathrm{min}$. The failure modes of triaxial CU shear tests on cement-soil core samples of D-1 and D-8 are shown in Figure 3(a). The stress-strain curves are shown in Figure 3(b). From the figure and the table we can see that:

(1)Cement-soil core samples are of brittle shear failure in the triaxial CU tests with a clear surface of shear failure. The angle between the surface of shear failure and the surface of the major principal stress is $60^{\circ} \sim 70^{\circ}$. And the stress-strain relations present strain softening. The failure strains were at a range from $0.63 \%$ to $1.83 \%$. Comparing with unconfined compressive tests, we can analyze that the effect of confining pressure makes the failure mode of core samples change from brittle destruction brittle vertical tension failure into brittle shear failure in inclined section, and also makes the failure strain increase.

(2)Like the results of unconfined compressive tests, there are some differences among the results of the unconfined compressive tests on cement-soil core samples with the same stake mark, including great difference and among results from D-6 to D-8, whose data are of larger discreteness.

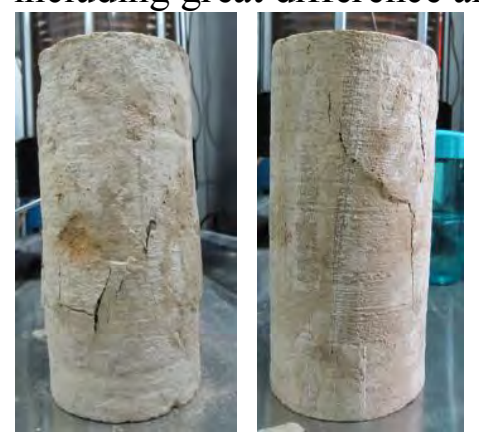

(a) Triaxial CU Shear Tests $(\sigma 3=100 \mathrm{kPa})$

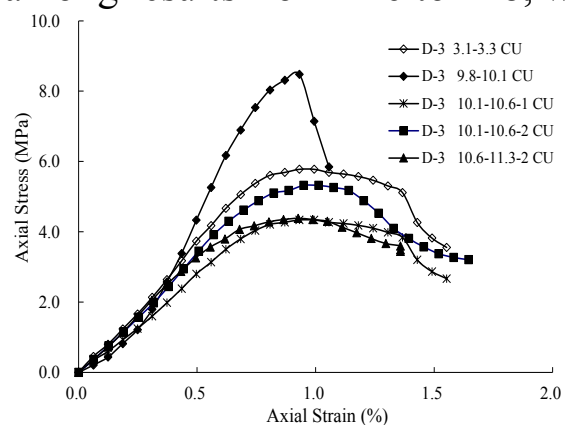

(b) The Stress-strain Curves

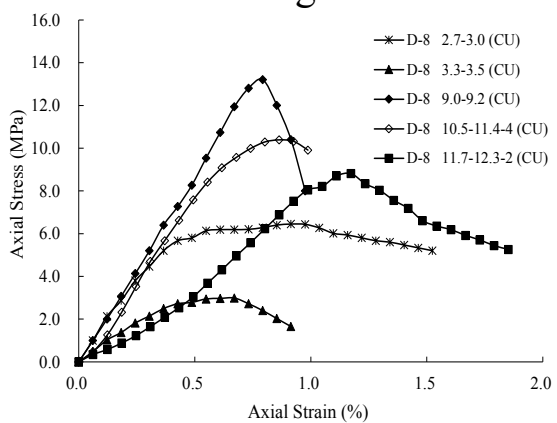

Fig. 3 Triaxial CU Shear Tests $\left(\sigma_{3}=100 \mathrm{kPa}\right)$

\section{Analyses of Test Results}

On the basis of the discreteness among the results of unconfined compressive tests and triaxial CU tests on cement-soil core samples with the same stake mark, the paper performs a statistical analysis on the test results referring to Code for investigation of geotechnical engineering (GB 50021-2009) and then obtains variable coefficients and standard values of cement-soil core samples with the same stake mark. The result showed that:

(1)Results of indoor strength tests are of good reliability. Variable coefficients are the important indicators on evaluating the homogeneity of the piles. The variable coefficients of unconfined compressive test results of D-1 D-4 and D-5 are in a range from 0.171 to 0.297 . The variable coefficients of unconfined compressive test results of D-6 D-8 and D-5 are in a range from 0.454 to 0.551 , It shows the heterogeneity of piles that the variable coefficients of D- $6 \sim$ D- 8 are too large and of which the results are of large discreteness. The discreteness of test results of D-1 D-4, D-5 is less so the homogeneity is better than that of D $-6 \sim \mathrm{D}-8$. When performing the analysis on test results this paper selects those test data with less discreteness. They are the data of D-1 D -4 and D-5.

(2)Use $0.94 \mathrm{~m} / \mathrm{min}$ as the lifting rate of D-4 and D-6 D-8 and $0.59 \mathrm{~m} / \mathrm{min}$ as the lifting rate of D-1 $\sim$ D-3 and D-5. It shows that the homogeneity of cement mixing piles with lower rate is better. 
Therefore, the lifting rate has a great impact on the construction quality of cement mixing piles. The lager the lifting rate is, the worse the homogeneity of mixing sand and cement is. So it is better to choose $0.59 \mathrm{~m} / \mathrm{min}$ as the lifting rate.

(3)Comparing the results of D-1 to that of D-2, and the results of D-3 to that of D-5, it shows that under the condition that when the cement mixing ratio is the same, the standard values of strength in unconfined compressive tests and triaxial CU tests on cement-soil core samples were greater when the water cement ratio of 1.0 than those when the water cement ratio is 0.8 . It indicates that the strength of cement-soil core samples is higher when the water cement ratio of 1.0 than that when the water cement ratio is 0.8 . And the higher the water cement ratio is, the larger the flowability of cement slurry is. Therefore the paper suggests selecting1.0 as the water cement ratio of construction.

(4)Comparing the results of D-1 to that of D-3, and the results of D-2 to that of D-5, it shows that under the condition that when the cement mixing ratio is the same, the higher the cement ratio is, the higher the strength of cement-soil core samples is. When the lifting ratio is $0.59 \mathrm{~m} / \mathrm{min}$ and the water cement ratio is 1.0 , they all have high strengths. In the project economic and appropriate cement mixing ratio should be selected according to the practical requirements and taking the requirement of the deformation property into account.

(5)The higher the strength is, the higher its modulus is correspondingly. For this kind of material like cement soil, the higher the modulus is, the higher the corresponding stress responds when a certain strain occurs and the more likely material reaches brittle failure. So in the project it is expected to reduce the modulus of the material as much as possible under conditions that can ensure the strength. Therefore the conception of modulus strength ratio (the ratio of modulus and strength) is proposed. The less modulus strength ratio is, the better the toughness of the material and the ability to adapt to the deformation are. When the lifting rate is $0.59 \mathrm{~m} / \mathrm{min}$, the modulus strength ratios of D-1 D-8 are from 70 to 130 . The modulus strength ratio of cement mixing pile is generally from 100 to 200 according to Technical code for ground treatment of buildings (JGJ79-2002). Under conditions that can ensure the strength to meet engineering requirements, it is beneficial to engineering safety to choose lower modulus strength ratio. Furthermore, the modulus is also an important indicator of calculating the deformation of the pile.

\section{Conclusion}

Through the experimental study on mechanical properties of cement-soil core samples in sand layer, the following conclusions and recommendations can be summarized:

(1)The lifting rate has a great impact on the homogeneity of the piles. Economic and appropriate lifting rate should be selected to guarantee the homogeneity of the piles. This study selects $0.59 \mathrm{~m} / \mathrm{min}$ as the better one.

(2)Under the condition that when the cement mixing ratio is the same, the standard values of strength in unconfined compressive tests and triaxial CU tests on cement-soil core samples were all higher when the water cement ratio of 1.0 than those when the water cement ratio is 0.8 . Considering the convenience of construction, it is more optimal to choose 1.0 as the water cement ratio.

(3) When the lifting ratio is $0.59 \mathrm{~m} / \mathrm{min}$ and the water cement ratio is 1.0 , the strengths in unconfined compressive tests are $2.499 \mathrm{MPa}$ and $4.028 \mathrm{MPa}$ for the cement ratio of $15 \%$ and $20 \%$ respectively. In the project economic and appropriate cement mixing ratio should be selected according to the practical requirements and following the principle of strength reaching the standard and the lower modulus strength ratio being superior.

\section{Acknowledgment}

This research is financially supported by National Natural Science Foundation of China (Grant No. 51378403 and No. 51309028). 


\section{References}

[1]GU Ming-fen, LIU Song-yu, HONG Zhen-yao, et al. Quantifying research on structural characteristics of cemented soils[J] Rock and Soil Mechanics, 2005, 26(11):1862-1868. (In Chinese)

[2]CHEN Su, CHEN Guo-xing. Research on composite foundation of cement pile [J] Journal of China \& Foreign Highway, 2007, 27(3):34-41. (In Chinese)

[3]ZENG Sheng-hua, ZENG Juan. Study on strength properties of low dosage cemented soil [J]. Subgrade Engineering, 2010(4): 17-20. (In Chinese)

[4]ZHOU Li-ping, SHEN Xiang-dong. Study on Mechanical Behaviors of cemented soil [J] Bulletin of the Chinese Ceramic Society, 2009, 28(2): 359-365. (In Chinese)

[5]LIU Xin, FAN Xiao-qiu, HONG Bao-ning. Experimental study of triaxial test of soils stabilized by cement mortar [J] Rock and Soil Mechanics, 2011, 32(6):1676-1680. (In Chinese)

[6]LIANG Ren-wang, ZHANG Ming, BAI Xiao-hong. Analysis of laboratory test results of cemented soil [J] Rock and Soil Mechanics, 2001, 22(2):211-213. (In Chinese)

[7]LI Jian-jun, LIANG Ren-wang. Research on compression strength and modulus of deformation of cemented soil[J] Rock and Soil Mechanics, 2009, 30(2):473-477. (In Chinese)

[8]XI Pei-sheng, LIU Song-yu, ZHANG Ba-fang. Electrical resistivity evaluation method to homogeneity of soil-cement deep mixing column [J] Journal of Southeast University (Natural Science Edition), 2007, 37(2):355-358. (In Chinese)

[9]DENG Yong-feng, LIU Song-yu, HONG Zhen-shun. Disturbance degree of surrounding soil induced by deep mixing column installation [J] Rock and Soil Mechanics, 2009, 30(3):717-720. (In Chinese)

[10]ZHANG Yi, CAO Zhi-guo, ZHANG Ding-wen. Statistical Analysis on Variability of Strength of Cement-soil [J] Subgrade Engineering, 2013(5):48-52. (In Chinese)

[11]AI Zhi-wei, LUO Si-hai, ZENG Yong, et al. Comparative study of cement-soil strength in the indoor and outdoor experiments[J] Journal of Jiangxi University of Science and Technology, 2013,34(3):47-53. (In Chinese)

[12]XU Song, YAN Chang-hong, XIA Wen-jun, et al. Comparing the results of indoor and core sample experiments with cement-soil strength[J] Journal of Geological Hazards and Environment Preservation, 2012, 23(3):64-67. (In Chinese)

[13]Former ministry of electric power industry of China. GB/T 50266-1999 Standard for test methods of engineering rock mass [S]. Beijing: Ministry of Construction of China, 1999. 